\title{
3D POINT CLOUD ENHANCEMENT USING GRAPH-MODELLED MULTIVIEW DEPTH MEASUREMENTS
}

\author{
Xue Zhang ${ }^{\star}$, Gene Cheung ${ }^{\star}$, Jiahao Pang ${ }^{\dagger}$, Dong Tian ${ }^{\dagger}$ \\ ${ }^{\star}$ Dept of EECS, York University, Toronto, Canada \\ $\dagger$ InterDigital, Princeton, New Jersey, USA
}

\begin{abstract}
A 3D point cloud is often synthesized from depth measurements collected by sensors at different viewpoints. The acquired measurements are typically both coarse in precision and corrupted by noise. To improve quality, previous works denoise a synthesized 3D point cloud a posteriori after projecting the imperfect depth data onto $3 \mathrm{D}$ space. Instead, we enhance depth measurements on the sensed images a priori, exploiting inherent 3D geometric correlation across views, before synthesizing a $3 \mathrm{D}$ point cloud from the improved measurements. By enhancing closer to the actual sensing process, we benefit from optimization targeting specifically the depth image formation model, before subsequent processing steps that can further obscure measurement errors. Mathematically, for each pixel row in a pair of rectified viewpoint depth images, we first construct a graph reflecting inter-pixel similarities via metric learning using data in previous enhanced rows. To optimize left and right viewpoint images simultaneously, we write a non-linear mapping function from left pixel row to the right based on 3D geometry relations. We formulate a MAP optimization problem, which, after suitable linear approximations, results in an unconstrained convex and differentiable objective, solvable using fast gradient method (FGM). Experimental results show that our method noticeably outperforms recent denoising algorithms that enhance after 3D point clouds are synthesized.
\end{abstract}

Index Terms - 3D point cloud, graph signal processing, graph learning, convex optimization

\section{INTRODUCTION}

Point Cloud (PC) is a signal representation composed of discrete geometric samples of a physical object in 3D space, useful for a range of imaging applications such as immersive communication and virtual / augmented reality (AR/VR) $[1,2]$. With recent advance and ubiquity of inexpensive active sensing technologies like Microsft Kinect and Intel RealSense, one method to generate a PC is to deploy multiple depth sensors to capture depth measurements (in the form of images) of an object from different viewpoints, then project these measurements to 3D space to synthesize a PC [3, 4]. Limitations in the depth acquisition process mean that the acquired depth measurements suffer from both imprecision and additive noise. This results in a noisy synthesized PC, and previous works focus on denoising PCs using a variety of methods: low-rank prior, low-dimensional manifold model (LDMM), surface smoothness priors expressed as graph total variation (GTV), graph Laplacian regularizer (GLR), feature graph Laplacian regularizer (GFLR), etc [5, 6, 7].

However, all the aforementioned denoising methods enhance a PC a posteriori, i.e., after a PC is synthesized from corrupted depth measurements. Recent work in image denoising $[8,9]$ has shown that by denoising raw sensed RGB measurements directly on the Bayer-patterned grid before demosaicking, contrast boosting and other steps typical in an image construction pipeline $[10,11]$ that obscure acquisition noise, one can dramatically improve the denoising performance compared to denoising on the image constructed after the pipeline (up to $15 \mathrm{~dB}$ in PSNR). Inspired by these work, we propose to enhance ${ }^{1}$ measurements in acquired depth images a priori, before projection to synthesize a PC. In our case, by enhancing closer to the actual physical sensing process before various steps in a PC synthesis pipeline including registration, stitching and filtering, we benefit from optimization that directly targets our depth-sensorspecific image formation model with a finite-bit pixel representation.

Specifically, towards a graph-smoothness signal prior [12, 13, 14], for each pixel row in a pair of rectified viewpoint depth images, we first construct a sparse graph reflecting inter-pixel similarities via metric learning [7] using data in previous enhanced rows. To exploit inter-view correlation and optimize left and right viewpoint images simultaneously, we write a non-linear mapping function from the left pixel row to the right based on 3D geometry relations. Using a depth image formation model that accounts for both additive noise and quantization, we formulate a maximum a posteriori (MAP) optimization problem, which, after suitable linear approximations, results in an unconstrained convex and differentiable objective, solvable using fast gradient method (FGM) [15]. Experimental results show that by enhancing measurements at the depth image level, our method outperforms several recent PC denoising algorithms [16, 17, 18] in two commonly used PC error metrics [19]. Related Work: Previous work on depth image enhancement [20, 21, 22] typically enhances one depth map at a time using image-based signal priors. When given two (or more) viewpoint depth maps, by ignoring the inherent cross-correlation between the views and optimizing each separately, the resulting quality is sub-optimal. One exception is [23], which considers noiseless but quantized observations per pixel from two views as signals in specified quantization bins. To reconstruct, the most likely signal inside both sets of quantization bins is chosen. Our work differs from [23] in that our image formation model considers both additive noise and quantization, leading to a more challenging MAP problem involving likelihood and prior terms from both views. We address this using appropriate linear approximations and FGM.

\section{SYSTEM OVERVIEW}

We assume a capturing system where the same 3D object is observed by two consumer-level depth cameras from different viewpoints, separated by distance $D$. Specifically, there exist overlapping fields of view $(\mathrm{FoV})$ from the two cameras, so that there are multiple

\footnotetext{
${ }^{1}$ We call our processing an "enhancement" that performs joint denoising and dequantization based on our depth image formation model.
} 


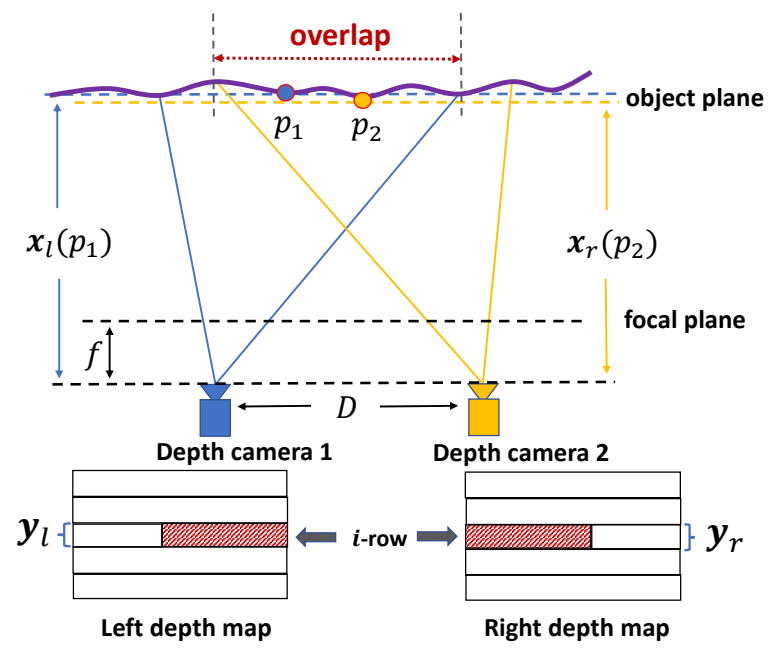

Fig. 1. An example of the camera system.

observations of the same 2D object surface. See Fig. 1 for an illustration. Each depth camera returns as output a depth map of resolution $H \times W$ and finite precision: each pixel is a noise-corrupted observation of the physical distance between the camera and the object, quantized to a $B$-bit representation. Without access to the underlying hardware pipeline, we assume that the depth map is the "rawest" signal we can acquire from the sensor.

For simplicity, we assume that the two captured depth maps are rectified; i.e., pixels in a row $i$ in the left view are capturing the same horizontal slice of the object as pixels in the same row $i$ in the right view. Rectification is a well-studied computer vision problem, and a known procedure [24] can be executed as a pre-processing step prior to our enhancement algorithm.

\section{PROBLEM FORMULATION}

We first describe a depth-sensor-specific image formation model and a mapping from left-view pixels to right-view pixels. We next define likelihood term and signal prior for depth images. Finally, we formulate a MAP optimization problem to enhance multiview depth measurements.

\subsection{Image Formation Model}

Denote by $\mathbf{y}_{l} \in \mathbb{R}^{N}\left(\mathbf{y}_{r} \in \mathbb{R}^{N}\right)$ an observed depth pixel row $i$ in the left (right) view. See Fig. 1 for details. (We forego index $i$ in $\mathbf{y}_{l}$ in the sequel for notation simplicity.) Observed pixels are noise-corrupted and quantized versions of the original depth measurements, $\mathbf{x}_{l}^{o}$ and $\mathbf{x}_{r}^{o}$, respectively. Specifically, observation $\mathbf{y}_{l}$ and true signal $\mathbf{x}_{l}^{o}$ are related via the following formation $\operatorname{model}^{2}$ :

$$
\mathbf{y}_{l}=\operatorname{round}\left(\frac{\mathbf{x}_{l}^{o}+\mathbf{n}_{l}}{Q}\right) Q
$$

where $Q \in \mathbb{R}^{+}$is a quantization parameter, and $\mathbf{n}_{l} \in \mathbb{R}^{N}$ is a zeromean additive noise. The same formation model applies for the right view. The goal is to optimally reconstruct signal $\widetilde{\mathbf{X}}=\left\{\widetilde{\mathbf{x}}_{l}, \widetilde{\mathbf{x}}_{r}\right\}$ given observation $\mathbf{Y}=\left\{\mathbf{y}_{l}, \mathbf{y}_{r}\right\}$.

\footnotetext{
${ }^{2}$ Practical quantization of depth measurements into $B$-bit representation for existing sensors are often non-uniform: larger depth distances are quantized into larger quantization bins. For simplicity, we model uniform quantization here, but the non-uniform generalization is straightforward.
}

\subsection{View-to-view Mapping}

Pixel rows $i$ of the rectified left and right views, $\mathbf{x}_{l}$ and $\mathbf{x}_{r}$, are projections from the same 2D object surface onto two different camera planes, and thus are related. For simplicity, we assume that there is no occlusion when projecting a $3 \mathrm{D}$ object to the two camera views. We employ a known 1D warping procedure [25] to relate $\mathbf{x}_{l}$ and $\mathbf{x}_{r}$. For the $j$-th pixel in the left view, $x_{l, j}$, its (non-integer) horizontal position $s\left(j, x_{l, j}\right)$ in the right view after projection is

$$
\begin{aligned}
& s\left(j, x_{l, j}\right)=j-\delta\left(x_{l, j}\right) \\
& \delta\left(x_{l, j}\right)=\frac{f D}{x_{l, j}}
\end{aligned}
$$

where $\delta()$ is the disparity, and $f$ is the camera focal length. Note that $s$ is a function of both left pixel's integer horizontal position $j$ and depth value $x_{l, j}$.

Assuming that the object surface is smooth, we interpolate right pixel row $\mathbf{x}_{r}$ given left pixel row $\mathbf{x}_{l}$ as

$$
\mathbf{x}_{r}=\mathbf{W}\left(\mathbf{x}_{l}\right) \mathbf{x}_{l}=\mathbf{g}\left(\mathbf{x}_{l}\right)
$$

where $\mathbf{W}\left(\mathbf{x}_{l}\right) \in \mathbb{R}^{N \times N}$ is a real weight matrix. (3) states that $\mathbf{x}_{r}$ is linearly interpolated from $\mathbf{x}_{l}$ using weights $\mathbf{W}\left(\mathbf{x}_{l}\right)$, where $\mathbf{W}\left(\mathbf{x}_{l}\right)$ is a function of signal $\mathbf{x}_{l}$. In particular, we model the weight $\omega_{i j}$ between right pixel $x_{r, i}$ and left pixel $x_{l, j}$ as

$$
\omega_{i j}=\frac{1}{\sum_{m=1}^{N} \exp \left(-\frac{\left(s\left(m, x_{l, m}\right)-i\right)^{2}}{\sigma_{s}^{2}}\right)} \exp \left(-\frac{\left(s\left(j, x_{l, j}\right)-i\right)^{2}}{\sigma_{s}^{2}}\right)
$$

In words, weight $\omega_{i j}$ is larger if the distance between the projected position $s\left(j, x_{l, j}\right)$ of left pixel $j$ and the target pixel position $i$ in $\mathbf{x}_{r}$ is small. To simplify (4), we assume a constant $C_{i}=1 / \sum_{m=1}^{N} \exp \left(-\frac{\left(s\left(m, x_{l, m}\right)-i\right)^{2}}{\sigma_{s}^{2}}\right)$. Combining with (2), (4) is rewritten as

$$
\omega_{i j}=C_{i} \exp \left(-\frac{\left(j-f D x_{l, j}^{-1}-i\right)^{2}}{\sigma_{s}^{2}}\right)
$$

Since $\mathbf{g}\left(\mathbf{x}_{l}\right)$ is differentiable, we use the first-order Taylor series expansion around $\mathbf{x}_{l}=\mathbf{x}_{l}^{0}$ to get a linear approximation, where $\mathbf{x}_{l}^{0}$ is the first estimate. Thus,

$$
\begin{aligned}
\mathbf{g}\left(\mathbf{x}_{l}\right) & \approx \mathbf{g}\left(\mathbf{x}_{l}^{0}\right)+\mathbf{g}^{\prime}\left(\mathbf{x}_{l}^{0}\right)\left(\mathbf{x}_{l}-\mathbf{x}_{l}^{0}\right) \\
& =\mathbf{g}^{\prime}\left(\mathbf{x}_{l}^{0}\right) \mathbf{x}_{l}+\mathbf{g}\left(\mathbf{x}_{l}^{0}\right)-\mathbf{g}^{\prime}\left(\mathbf{x}_{l}^{0}\right) \mathbf{x}_{l}^{0} \\
& =\mathbf{H} \mathbf{x}_{l}+\mathbf{d}
\end{aligned}
$$

where $\mathbf{H}=\mathbf{g}^{\prime}\left(\mathbf{x}_{l}^{0}\right)=\left[\frac{\partial g_{i}\left(\mathbf{x}_{l}^{0}\right)}{\partial x_{l, j}}\right]_{i, j} \in \mathbb{R}^{N \times N}$ is the Jacobian matrix (first-order partial derivatives) of $\mathbf{g}\left(\mathbf{x}_{l}\right)$ at $\mathbf{x}_{l}=\mathbf{x}_{l}^{0}$, and $\mathbf{d}=\mathbf{g}\left(\mathbf{x}_{l}^{0}\right)-\mathbf{g}^{\prime}\left(\mathbf{x}_{l}^{0}\right) \mathbf{x}_{l}^{0}$ is a constant vector.

\subsection{Likelihood Term}

We assume that the zero-mean additive noise $\mathbf{n}_{l} \in \mathbb{R}^{N}$ follows a jointly Gaussian distribution; i.e., the probability density function (pdf) of $\mathbf{n}_{l}$ is

$$
\operatorname{Pr}\left(\mathbf{n}_{l}\right)=\exp \left(-\frac{\mathbf{n}_{l}^{\top} \mathbf{P}_{l} \mathbf{n}_{l}}{\sigma_{n}^{2}}\right)
$$




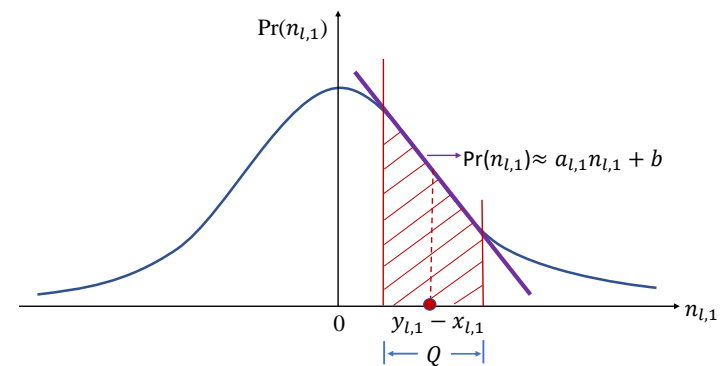

Fig. 2. Affine approximation of the Gaussian pdf.

where $\mathbf{P}$ is a positive definite (PD) precision matrix, and $\sigma_{n}^{2}$ is the noise variance. Given observation $\mathbf{y}_{l}$, the likelihood term $\operatorname{Pr}\left(\mathbf{y}_{l} \mid \mathbf{x}_{l}\right)$ is

$$
\operatorname{Pr}\left(\mathbf{y}_{l} \mid \mathbf{x}_{l}\right)=\int_{\mathcal{R}_{l}} \operatorname{Pr}\left(\mathbf{n}_{l}\right) d \mathbf{n}_{l}
$$

where the region $\mathcal{R}_{l}$ over which the integration (10) is performed is defined as

$$
\mathcal{R}_{l}=\left\{\mathbf{n}_{l, i} \mid \mathbf{y}_{l, i}-\frac{Q}{2}<\mathbf{x}_{l, i}+\mathbf{n}_{l, i}<\mathbf{y}_{l, i}+\frac{Q}{2}\right\}
$$

We assume that left and right noise $\mathbf{n}_{l}$ and $\mathbf{n}_{r}$ are independent.

The integration in (10) over a jointly Gaussian pdf is non-trivial. Instead, we first approximate $\operatorname{Pr}\left(\mathbf{n}_{l}\right)$ over the region $\mathcal{R}_{l}$ as an affine function

$$
\operatorname{Pr}\left(\mathbf{n}_{l}\right) \approx \mathbf{a}^{\top} \mathbf{n}_{l}+b
$$

where contants $\mathbf{a} \in \mathbb{R}^{N}$ and $b \in \mathbb{R}$ can be computed via Taylor series expansion at $\mathbf{y}_{l}-\mathbf{x}_{l}$ given (9). See 1-D case in Fig. 2 for an illustration. For reasonably small $Q$, this is a good approximation. We now rewrite (10) as

$$
\begin{aligned}
\operatorname{Pr}\left(\mathbf{y}_{l} \mid \mathbf{x}_{l}\right) & \approx \int_{\mathcal{R}_{l}}\left(\mathbf{a}^{\top} \mathbf{n}_{l}+b\right) d \mathbf{n}_{l} \\
& =Q^{N}\left(\mathbf{a}^{\top}\left(\mathbf{y}_{l}-\mathbf{x}_{l}\right)+b\right)
\end{aligned}
$$

where (14) is proven in the Appendix.

\subsection{Signal Prior}

As done in recent graph-based image processing work [26, 27, 28], we model the similarities among pixels in $\mathbf{x}_{l}$ using a graph Laplacian matrix $\mathbf{L}_{l}$, and thus prior $\operatorname{Pr}\left(\mathbf{x}_{l}\right)$ can be written as:

$$
\operatorname{Pr}\left(\mathbf{x}_{l}\right)=\exp \left(-\frac{\mathbf{x}_{l}^{\top} \mathbf{L}_{l} \mathbf{x}_{l}}{\sigma^{2}}\right)
$$

We assume that the previous $K$ pixel rows in the left depth image have been enhanced, and assuming in addition that the next row $i$ follows a similar image structure, $\mathbf{L}_{l}$ can be learned from the previous $K$ rows. See Section 4 for details.

\subsection{MAP Formulation}

We now formulate a MAP problem for $\widetilde{\mathbf{X}}=\left\{\widetilde{\mathbf{x}}_{l}, \widetilde{\mathbf{x}}_{r}\right\}$ as follows.

$$
\begin{aligned}
& \max _{\mathbf{x}_{l}, \mathbf{x}_{r}} \operatorname{Pr}\left(\mathbf{y}_{l}, \mathbf{y}_{r} \mid \mathbf{x}_{l}, \mathbf{x}_{r}\right) \operatorname{Pr}\left(\mathbf{x}_{l}, \mathbf{x}_{r}\right) \\
& =\operatorname{Pr}\left(\mathbf{y}_{l}, \mathbf{y}_{r} \mid \mathbf{x}_{l}, \mathbf{g}\left(\mathbf{x}_{l}\right)\right) \operatorname{Pr}\left(\mathbf{x}_{l}, \mathbf{g}\left(\mathbf{x}_{l}\right)\right) \\
& =\operatorname{Pr}\left(\mathbf{y}_{l} \mid \mathbf{x}_{l}\right) \operatorname{Pr}\left(\mathbf{y}_{r} \mid \mathbf{g}\left(\mathbf{x}_{l}\right)\right) \operatorname{Pr}\left(\mathbf{x}_{l}\right) \operatorname{Pr}\left(\mathbf{g}\left(\mathbf{x}_{l}\right)\right) \\
& \approx Q^{2 N}\left(\mathbf{a}^{\top}\left(\mathbf{y}_{l}-\mathbf{x}_{l}\right)+b\right)\left(\mathbf{a}^{\top}\left(\mathbf{y}_{r}-\mathbf{g}\left(\mathbf{x}_{l}\right)\right)+b\right) \\
& \exp \left(-\frac{\mathbf{x}_{l}^{\top} \mathbf{L}_{l} \mathbf{x}_{l}}{\sigma^{2}}\right) \exp \left(-\frac{\mathbf{g}\left(\mathbf{x}_{l}\right)^{\top} \mathbf{L}_{r} \mathbf{g}\left(\mathbf{x}_{l}\right)}{\sigma^{2}}\right)
\end{aligned}
$$

where in (17) we substituted $\mathbf{g}\left(\mathbf{x}_{l}\right)$ for $\mathbf{x}_{r}$, and in (18) we split up the first term since left and right noise, $\mathbf{n}_{l}$ and $\mathbf{n}_{r}$, are independent.

To ease optimization, we minimize the negative log of (19):

$$
\begin{aligned}
\min _{\mathbf{x}_{l}} & -\ln \left(\mathbf{a}^{\top}\left(\mathbf{y}_{l}-\mathbf{x}_{l}\right)+b\right)-\ln \left(\mathbf{a}^{\top}\left(\mathbf{y}_{r}-\mathbf{g}\left(\mathbf{x}_{l}\right)\right)+b\right) \\
& +\mathbf{x}_{l}^{\top} \mathbf{L}_{l} \mathbf{x}_{l}+\mathbf{g}\left(\mathbf{x}_{l}\right)^{\top} \mathbf{L}_{r} \mathbf{g}\left(\mathbf{x}_{l}\right) \\
= & -\ln \left(\mathbf{a}^{\top}\left(\mathbf{y}_{l}-\mathbf{x}_{l}\right)+b\right)-\ln \left(\mathbf{a}^{\top}\left(\mathbf{y}_{r}-\left(\mathbf{H} \mathbf{x}_{l}+\mathbf{d}\right)\right)+b\right) \\
& +\mathbf{x}_{l}^{\top} \mathbf{L}_{l} \mathbf{x}_{l}+\mathbf{x}_{l}^{\top} \mathbf{H}^{\top} \mathbf{L}_{r} \mathbf{H} \mathbf{x}_{l}+2 \mathbf{d}^{\top} \mathbf{L}_{r} \mathbf{H} \mathbf{x}_{l}+\mathbf{d}^{\top} \mathbf{L}_{r} \mathbf{d}
\end{aligned}
$$

(21) is an unconstrained convex and differentiable objective; we can solve for its minimum efficiently using FGM.

\section{FEATURE GRAPH LEARNING}

\subsection{Learning Metric for Graph Construction}

When pixel row $i$ of the left view is optimized, we assume that the previous $K$ rows, $i-1, \ldots i-K$, have already been enhanced into $\widetilde{\mathbf{x}}_{l}^{i-1}, \ldots \widetilde{\mathbf{x}}_{l}^{i-K}$. Using these $K$ enhanced rows, we compute graph Laplacian $\mathbf{L}_{l}$ to define prior $\operatorname{Pr}(\mathbf{x})$ in (15). Because in a practice $K<N$, estimating $\mathbf{L}_{l} \in \mathbb{R}^{N \times N}$ reliably using only $K$ signal observations is a known difficult small data learning problem. In particular, established graph learning algorithms such as graphical LASSO [29] and constrained $l_{1}$-norm minimization (CLIME) [30] that compute a sparse precision matrix using as input an accurate empirical covariance matrix estimated from a large number of observations do not work in our small data learning scenario.

Instead, inspired by [7] we construct an appropriate similarity graph via metric learning. We first assume that associated with each pixel (graph node) $i$ in $\mathbf{x}_{l}$ is a length- $F$ relevant feature vector $\mathbf{f}_{i} \in$ $\mathbb{R}^{F}$ (to be discussed). The feature distance $d_{i j}$ between two nodes $i$ and $j$ is computed using a real, symmetric and PD metric matrix $\mathbf{M} \in \mathbb{R}^{F \times F}$ as

$$
d_{i j}=\left(\mathbf{f}_{i}-\mathbf{f}_{j}\right)^{\top} \mathbf{M}\left(\mathbf{f}_{i}-\mathbf{f}_{j}\right)
$$

Since $\mathbf{M}$ is $\mathrm{PD}, d_{i j}>0$ for $\mathbf{f}_{i}-\mathbf{f}_{j} \neq \mathbf{0}$. The edge weight $w_{i j}$ between nodes $i$ andd $j$ is then computed using a Gaussian kernel:

$$
w_{i j}=\exp \left(-d_{i j}\right)
$$

To optimize M, we minimize the graph Laplacian regularizer (GLR) evaluated using $K$ previous pixel rows:

$$
\begin{aligned}
\min _{\mathbf{M} \succ 0} & \sum_{k=1}^{K}\left(\widetilde{\mathbf{x}}_{l}^{k}\right)^{\top} \mathbf{L}_{l}^{k}(\mathbf{M}) \widetilde{\mathbf{x}}_{l}^{k} \\
& =\sum_{k=1}^{K} \sum_{i, j} w_{i j}^{k}\left(\widetilde{x}_{l, i}^{k}-\widetilde{x}_{l, j}^{k}\right)^{2}
\end{aligned}
$$

where edge weights $w_{i j}^{k}$ in Laplacian $\mathbf{L}_{l}^{k}(\mathbf{M})$ is computed using features $\mathbf{f}_{i}^{k}$ and $\mathbf{f}_{j}^{k}$ of the $k$-th observation $\widetilde{\mathbf{x}}_{l}^{k}$ and equations (22) and (23). To optimize $\mathbf{M}$ in (24), [7] proposed a fast optimization algorithm to optimize the diagonal and off-diagonal entries of $\mathbf{M}$ alternately. See [7] for details.

\subsection{Feature Selection for Metric Learning}

To construct a feature vector $\mathbf{f}_{i}$ for each pixel $i$ in $\mathbf{x}_{l}$, we first compute the pixel's corresponding surface normal $\mathbf{n}_{i} \in \mathbb{R}^{3}$ by projecting it to $3 \mathrm{D}$ space and computing it using its neighboring points via 
Table 1. C2C and C2P results of competing methods at three noise levels

\begin{tabular}{|c|c|c|c|c|c|c|}
\hline$\sigma_{n}^{2}$ & methods & Adirondack & ArtL & Teddy & Recycle & Playtable \\
\hline \multirow{8}{*}{50} & & 3.63 & 3.47 & 2.76 & 3.92 & 4.08 \\
\hline & APSS & $14.45^{\circ}$ & 11.73 & 7.26 & 15.79 & 17.42 \\
\hline & & 3.47 & 3.35 & 2.67 & 3.72 & 4.09 \\
\hline & RIMLS & 13.26 & 11.21 & 7.09 & 15.11 & 17.06 \\
\hline & MRPCA & 2.91 & 3.05 & 2.55 & 3.17 & 3.21 \\
\hline & ИККСА & 8.86 & 8.73 & 6.21 & 10.21 & 9.17 \\
\hline & Pronosed & 2.11 & 2.26 & 1.56 & 2.45 & 3.09 \\
\hline & Proposed & 4.88 & 6.34 & 2.79 & 7.00 & 8.78 \\
\hline \multirow{8}{*}{70} & & 4.12 & 3.80 & 3.09 & 4.34 & 4.46 \\
\hline & APSS & 18.56 & 13.88 & 8.96 & 20.07 & 18.91 \\
\hline & & 3.83 & 3.67 & 3.00 & 4.16 & 4.38 \\
\hline & RIMLS & $17.26^{\circ}$ & 13.41 & 8.73 & 19.42 & 18.92 \\
\hline & MRPCA & 3.42 & 3.45 & 2.89 & 3.76 & 3.48 \\
\hline & МKRLA & 12.57 & 11.39 & 7.98 & 14.80 & 11.00 \\
\hline & Proposed & 2.32 & 2.48 & 1.68 & 2.68 & 3.26 \\
\hline & & 5.97 & 7.64 & 3.32 & 8.47 & 10.43 \\
\hline \multirow{7}{*}{90} & APSS & 4.40 & 4.28 & 3.38 & 4.80 & 4.91 \\
\hline & & $21.97^{\circ}$ & 17.07 & 11.08 & 25.11 & 26.18 \\
\hline & RIMLS & $\begin{array}{l}4.19 \\
21.15\end{array}$ & $\begin{array}{l}4.13 \\
16.52\end{array}$ & 3.30 & $\begin{array}{l}4.59 \\
24.6\end{array}$ & $\begin{array}{l}4.83 \\
23.46\end{array}$ \\
\hline & & 3.78 & 3.91 & 3.20 & 4.20 & $\frac{23.40}{3.95}$ \\
\hline & MRPCA & 16.11 & 14.07 & 9.69 & 19.10 & 14.52 \\
\hline & Proposed & 2.47 & 2.70 & 1.84 & 2.92 & 3.45 \\
\hline & & 6.95 & 9.15 & 4.08 & 10.45 & 13.22 \\
\hline
\end{tabular}

method [31]. Then together with depth value $x_{i}$ and location $\mathbf{l}_{i} \in \mathbb{R}^{2}$ in the $2 \mathrm{D}$ grid, we construct $\mathbf{f}_{i} \in \mathbb{R}^{6}$. Because $\mathbf{M}$ is symmetric, the number of matrix entries we need to estimate is only 21 .

\section{EXPERIMENTS}

We conducted simulations with five depth image pairs provided in Middlebury datasets [32]: Adirondack, Recycle, Playtable, Teddy and ArtL. By projecting left and right views to 3D space, the first three generate PCs with around 700000 points, Teddy with 337500 points and ArtL with 192238 points. Gaussian noise with zero mean and variance $\sigma_{n}^{2}$ of 50,70 and 90 is added to both left and right views, which are then quantized into 256 distinct values. To compute the precision matrix $\mathbf{P}$ for noise in pixel row $i$, we use previous $K_{n}=30$ estimated noise terms to compute the covariance matrix, where $\widetilde{\mathbf{n}}^{i-k_{n}}=\mathbf{y}^{i-k_{n}}-\widetilde{\mathbf{x}}^{i-k_{n}}$ and $1 \leq k_{n} \leq K_{n}$. When learning metric for graph construction, we consider previous $K=10$ pixel rows. To reduce computation complexity, the same optimized $\mathbf{M}$ is used for the next $K$ pixel rows. Based on the feature vector in $\mathbf{y}^{i}$ of the current row $i$, we can finally compute the corresponding Laplacian $\mathbf{L}^{i}(\mathbf{M})$.

Our proposed 3D PC enhancement method is compared against three existing PC denoising algorithms: APSS [16], RIMLS [17] and the moving robust principle component analysis (MRPCA) algorithm [18]. APSS and RIMLS are implemented with MeshLab software, and the source code of MRPCA is provided by the authors. Two commonly used PC evaluation metrics, point-to-point $(\mathrm{C} 2 \mathrm{C})$ error and point-to-plane $(\mathrm{C} 2 \mathrm{P})$ error between ground truth and denoising point sets, are employed.

After projecting both noise-corrupted and quantized left and right views into a $\mathrm{PC}$, one can employ three mentioned $\mathrm{PC}$ denoising algorithms. $\mathrm{C} 2 \mathrm{C}$ and $\mathrm{C} 2 \mathrm{P}$ results of different methods with three noise levels are shown in Table 1. Overall, our method achieves by far the best performance in both metrics and all three noise levels, with $\mathrm{C} 2 \mathrm{C}$ reduced by $0.68,0.92,1.13$; and $\mathrm{C} 2 \mathrm{P}$ reduced by 2.68 , $4.38,5.93$ on average compared to the second best algorithm for $\sigma_{n}^{2}=50,70,90$, respectively.

Visual results for Recycle is shown in Fig. 3. For better visualization, we use CloudCompare software to show the $\mathrm{C} 2 \mathrm{C}$ absolute distances between the ground truth points and their closest denoised

points. We observe that our proposed method achieves smaller $\mathrm{C} 2 \mathrm{C}$ errors (in blue) compared to the competitors.

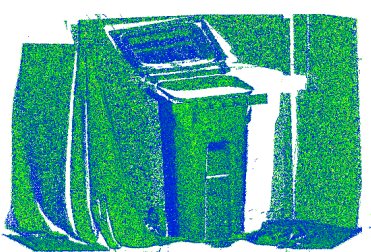

(a) APSS

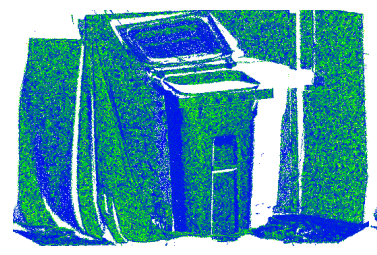

(c) MRPCA

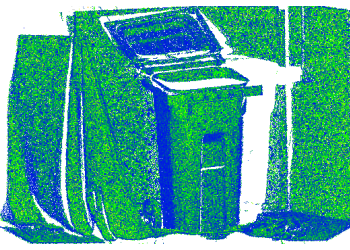

(b) RIMLS

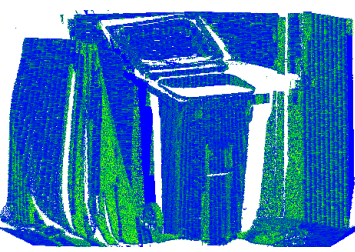

(d) Proposed

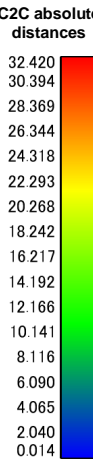

2.040
0.014

Fig. 3. Comparison of visual results for Recycle when $\sigma_{n}^{2}=50$. From blue to red, C2C absolute errors gradually become larger. More blue points are noticely included in the proposed method.

\section{CONCLUSION}

Point clouds are typically synthesized from finite-precision depth measurements that are noise-corrupted. In this paper, we improve the quality of a synthesized point cloud by jointly enhancing multiview depth images-the "rawest" signal we can acquire from an off-the-shelf sensor-prior to modules in a typical point cloud synthesis pipeline that obscure acquisition noise. We formulate a graphbased MAP optimization that specifically targets an image formation model accounting for both additive noise and quantization. Simulation results show that our proposed scheme outperforms competing schemes that denoise point clouds after the synthesis pipeline.

\section{A. PROOF OF MULTIPLE INTEGRAL}

We prove (14) by induction. Consider first the base case $(N=1)$ when $x_{l}, y_{l}, n_{l} \in \mathbb{R}$ and $\operatorname{Pr}\left(n_{l}\right) \approx a n_{l}+b$, where $a, b \in \mathbb{R}$. Integral in (10) in this case is a single integral, and one can easily check that $\operatorname{Pr}\left(y_{l} \mid x_{l}\right)=Q\left(a\left(y_{l}-x_{l}\right)+b\right)$. Consider next the inductive case and assume $\operatorname{Pr}\left(\mathbf{y}_{l} \mid \mathbf{x}_{l}\right)=Q^{N}\left(\mathbf{a}^{\top}\left(\mathbf{y}_{l}-\mathbf{x}_{l}\right)+b\right)$, when $\mathbf{x}_{l}, \mathbf{y}_{l}, \mathbf{n}_{l} \in \mathbb{R}^{N}$. If the dimension of the signal is actually $N+1$, when integrating the first $N$ variables, the $N+1$-th term $a_{N+1} n_{N+1}$ is treated the same as constant $b$, thus,

$\operatorname{Pr}\left(\mathbf{y}_{l} \mid \mathbf{x}_{l}\right)=\int_{z_{N+1}-Q / 2}^{z_{N+1}+Q / 2} Q^{N}\left(\mathbf{a}^{\prime \top} \mathbf{z}_{l}^{\prime}+b+a_{N+1} n_{N+1}\right) d n_{N+1}$

where $\mathbf{a}^{\prime}$ and $\mathbf{z}_{l}^{\prime}=\mathbf{y}_{l}-\mathbf{x}_{l}$ are vectors for only the first $N$ terms. Since $\mathbf{a}^{\prime \top} \mathbf{z}_{l}^{\prime}+b$ is constant, like the base case one can easily integrate this, resulting in

$$
\begin{aligned}
\operatorname{Pr}\left(\mathbf{y}_{l} \mid \mathbf{x}_{l}\right) & =Q^{N}\left[Q\left(a_{N+1} z_{N+1}+\mathbf{a}^{\prime \top} \mathbf{z}_{l}^{\prime}+b\right)\right] \\
& =Q^{N+1}\left(\mathbf{a}^{\top} \mathbf{z}_{l}+b\right)
\end{aligned}
$$

where $\mathbf{a}$ and $\mathbf{z}_{l}$ are vectors for all $N+1$ terms. 


\section{B. REFERENCES}

[1] M. Wien, J. M Boyce, T. Stockhammer, and W.-H. Peng, "Standardization status of immersive video coding," IEEE Journal on Emerging and Selected Topics in Circuits and Systems, vol. 9, no. 1, pp. 5-17, 2019.

[2] M. L. Steven, Virtual Reality, Cambridge University Press: Cambridge, UK, 2016.

[3] R. Hartley and A. Zisserman, Multiple view geometry in computer vision, Cambridge university press, 2003.

[4] X. Huang, X. Cheng, Q. Geng, B. Cao, D. Zhou, P. Wang, Y. Lin, and R. Yang, "The apolloscape dataset for autonomous driving," in Proceedings of the IEEE Conference on Computer Vision and Pattern Recognition Workshops, 2018, pp. 954-960.

[5] C. Dinesh, G. Cheung, I. V Bajić, and C. Yang, "Local 3D point cloud denoising via bipartite graph approximation \& total variation," in 2018 IEEE International Workshop on Multimedia Signal Processing. IEEE, 2018, pp. 1-6.

[6] J. Zeng, G. Cheung, M. Ng, J. Pang, and C. Yang, "3D point cloud denoising using graph laplacian regularization of a low dimensional manifold model," IEEE Transactions on Image Processing, vol. 29, pp. 3474-3489, 2019.

[7] W. Hu, X. Gao, G. Cheung, and Z. Guo, "Feature graph learning for 3D point cloud denoising," in arXiv preprint arXiv: 1907.09138, 2019.

[8] A. Punnappurath and M. S Brown, "Learning raw image reconstruction-aware deep image compressors," IEEE transactions on pattern analysis and machine intelligence, Early Access, 2019.

[9] R. M. Nguyen and M. S Brown, "Raw image reconstruction using a self-contained sRGB-JPEG image with only $64 \mathrm{~KB}$ overhead," in Proceedings of the IEEE Conference on Computer Vision and Pattern Recognition, 2016, pp. 1655-1663.

[10] S. Farsiu, M. Elad, and P. Milanfar, "Multiframe demosaicing and super-resolution of color images," IEEE transactions on image processing, vol. 15, no. 1, pp. 141-159, 2005.

[11] D. Sahu, A. Bhargava, and P. Badal, "Contrast image enhancement using various approaches: A review," Journal of Image Processing \& Pattern Recognition Progress, vol. 4, no. 3, pp. 39-45, 2017.

[12] A. Ortega, P. Frossard, J. Kovacevic, J. M. F. Moura, and P. Vandergheynst, "Graph signal processing: Overview, challenges, and applications," in Proceedings of the IEEE, May 2018, vol. 106, no.5, pp. 808-828.

[13] G. Cheung, E. Magli, Y. Tanaka, and M. Ng, "Graph spectral image processing," in Proceedings of the IEEE, May 2018, vol. 106, no.5, pp. 907-930.

[14] J. Pang and G. Cheung, "Graph Laplacian regularization for inverse imaging: Analysis in the continuous domain," in IEEE Transactions on Image Processing, April 2017, vol. 26, no.4, pp. 1770-1785.

[15] Y. Nesterov, Introductory lectures on convex optimization: A basic course, vol. 87, Springer Science \& Business Media, 2013.

[16] G. Guennebaud and M. Gross, "Algebraic point set surfaces," in ACM SIGGRAPH 2007 papers, pp. 23-es. 2007.
[17] A C. Öztireli, G. Guennebaud, and M. Gross, "Feature preserving point set surfaces based on non-linear kernel regression," in Computer Graphics Forum. Wiley Online Library, 2009, vol. 28, pp. 493-501.

[18] E. Mattei and A. Castrodad, "Point cloud denoising via moving rpca," in Computer Graphics Forum. Wiley Online Library, 2017, vol. 36, pp. 123-137.

[19] D. Tian, H. Ochimizu, C. Feng, R. Cohen, and A. Vetro, "Geometric distortion metrics for point cloud compression," in 2017 IEEE International Conference on Image Processing. IEEE, 2017, pp. 3460-3464.

[20] W. Hu, G. Cheung, and M. Kazui, "Graph-based dequantization of block-compressed piecewise smooth images," in IEEE Signal Processing Letters, February 2016, vol. 23, no.2, pp. 242-246.

[21] S. Gu, W. Zuo, S. Guo, Y. Chen, C. Chen, and L. Zhang, "Learning dynamic guidance for depth image enhancement," in Proceedings of the IEEE conference on computer vision and pattern recognition, 2017, pp. 3769-3778.

[22] J. Jeon and S. Lee, "Reconstruction-based pairwise depth dataset for depth image enhancement using CNN," in Proceedings of the European Conference on Computer Vision, 2018, pp. 422-438.

[23] P. Wan, G. Cheung, P. Chou, D. Florencio, C. Zhang, and $\mathrm{O}$. Au, "Precision enhancement of 3D surfaces from compressed multiview depth maps," in IEEE Signal Processing Letters, October 2015, vol. 22, no.10, pp. 1676-1680.

[24] C. Loop and Z. Zhang, "Computing rectifying homographies for stereo vision," in Proceedings of the IEEE Computer Society Conference on Computer Vision and Pattern Recognition (Cat. No PR00149). IEEE, 1999, vol. 1, pp. 125-131.

[25] J. Jin, A. Wang, Y. Zhao, C. Lin, and B. Zeng, "Region-aware 3-D warping for DIBR," IEEE Transactions on Multimedia, vol. 18, no. 6, pp. 953-966, 2016.

[26] V. Kalofolias, "How to learn a graph from smooth signals," in Artificial Intelligence and Statistics, 2016, pp. 920-929.

[27] H. E Egilmez, E. Pavez, and A. Ortega, "Graph learning from data under laplacian and structural constraints," IEEE Journal of Selected Topics in Signal Processing, vol. 11, no. 6, pp. 825841, 2017.

[28] Y. Bai, G. Cheung, X. Liu, and W. Gao, "Graph-based blind image deblurring from a single photograph," IEEE Transactions on Image Processing, vol. 28, no. 3, pp. 1404-1418, 2018.

[29] J. Friedman, T. Hastie, and R. Tibshirani, "Sparse inverse covariance estimation with the graphical lasso," in Biostatistics, 2008, vol. 9, no.3, pp. 432-441.

[30] T. Cai, W. Liu, and X. Luo, "A constrained $\ell_{1}$ minimization approach to sparse precision matrix estimation," in Journal of the American Statistical Association, 2011, vol. 106, pp. 594607.

[31] H. Avron, A. Sharf, C. Greif, and D. Cohen-Or, " $\ell_{1}$-sparse reconstruction of sharp point set surfaces," ACM Transactions on Graphics (TOG), vol. 29, no. 5, pp. 1-12, 2010.

[32] D. Scharstein, H. Hirschmüller, Y. Kitajima, G. Krathwohl, N. Nesic, X. Wang, and P. Westling, "High-resolution stereo datasets with subpixel-accurate ground truth," in German conference on pattern recognition. Springer, 2014, pp. 31-42. 